\title{
Raman Imaging Spectroscopy: History, Fundamentals and Current Scenario of the Technique
}

\author{
Hery Mitsutake, ${ }^{a}$ Ronei J. Poppi ${ }^{a}$ and Márcia C. Breitkreitz ${ }^{\circledR *, a}$ \\ ${ }^{a}$ Departamento de Química Analítica, Instituto de Química, \\ Universidade Estadual de Campinas (Unicamp), 13084-971 Campinas- SP, Brazil
}

\begin{abstract}
Raman imaging spectroscopy is a relatively new technique that has raised interest in several areas of knowledge due to the possibility of evaluating the spatial distribution of compounds based on their spectra. This paper presents a brief history of the development of this important technique, a theoretical description of the Raman effect and the preprocessing and processing (both univariate and multivariate) tools for Raman data. We also describe the current state of applications for major areas such as agriculture/biomass, environment, biology, medicine, pharmaceutical, geology, foodstuff, artwork, archaeology, material characterization and forensic.
\end{abstract}

Keywords: Raman imaging, Raman mapping, Raman spectroscopy, chemical imaging

\section{Introduction}

Raman spectroscopy is a technique within vibrational spectroscopy, which is based on the inelastic scattering of light. Since the development of the first commercial Raman spectrometer in 1953, advances in lasers and detectors and the discovery of new phenomena have expanded the use of this technique in several research fields.

In 1966, Delhaye and Migeon ${ }^{1}$ suggested that Raman scattering was independent of sample volume and therefore could be applied for microscopic particle analysis. ${ }^{1}$ From this concept, in 1974, the first Raman micro-spectrometers were developed and commercialized, which provided Raman point and imaging analysis., ${ }^{2,3}$ The gain obtained by combining spectral information with spatial information allowed this technique to be used to analyze various matrices, gaining prominence due to the quality of the generated results. The use of spectroscopic images allows evaluating the spatial distribution of the compounds based on their spectra, fundamental to understanding characteristics and chemical and/or physical properties related to the sample.

Raman imaging spectroscopy combines a microscope with a Raman spectrometer. Figure 1 shows a representation of the generated data, which is called a data cube or a data hypercube. Each square within the cube represents one pixel; the dimensions $\mathrm{M}$ and $\mathrm{N}$ represent the spatial

*e-mail: marciacb@unicamp.br dimensions, i.e., the coordinates/localizations of the pixels, while the dimension $\lambda$ represents the spectral dimension.

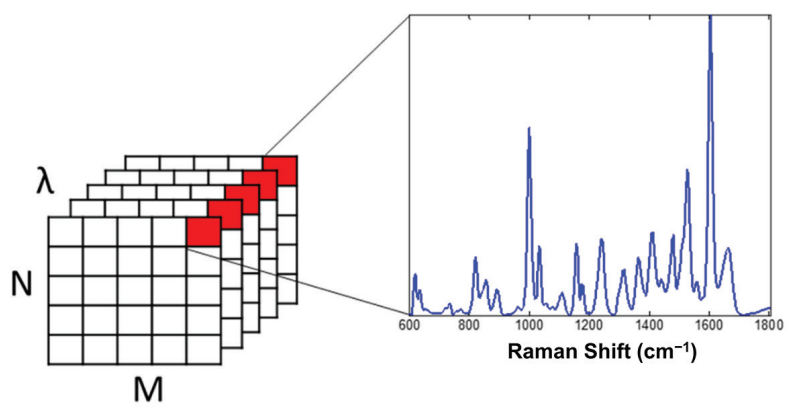

Figure 1. Data cube obtained by Raman imaging spectroscopy.

Imaging spectroscopy has filled an existing gap in relation to the physicochemical characterization of materials since other techniques of microscopy, such as scanning electron microscope (SEM) and transmission electron microscope (TEM), only generate information related to the morphology of the mapped surface. Notable exceptions are scanning electron microscopy coupled with energy dispersive X-ray spectroscopy (SEM/EDS) or fluorescence microscopy, which also provide chemical and spatial information about the analyzed area. In SEM/EDS, characteristic X-rays are generated by inelastic scattering of electrons using an incident beam. ${ }^{4}$ One of the great advantages of this technique is that it allows the identification and quantification of almost all elements of the periodic table (except for $\mathrm{H}, \mathrm{He}$ and $\mathrm{Li}$ ), with 
limits of detection typically in the range of 0.001-0.003 of mass fraction. ${ }^{5}$ However, one of the disadvantages of this technique is the need of different elements to extract chemical maps, i.e., the compounds must have one different element (at least) in their structures to be able to evaluate their distribution in the analyzed region. In the case of fluorescence microscopy, its main use is to analyze specimens that contain or were treated with fluorescent species, usually dyes. These species absorb energy at a given wavelength and emit light at other wavelengths with less energy (longer wavelength). With the dye having affinity for a particular compound, using fluorescence microscopy, it is possible to selectively examine this compound in the desired area. ${ }^{6}$

Over the last ten years, several advances have been made and the application of Raman spectroscopy imaging has increased over the time. In some areas, it is already used as a routine technique (for example, in material characterization), while in others, it has been explored as an alternative to traditional methods (e.g., in the medical or geologic fields). Due to the diversity of applications and techniques, the aim of this review is to provide an overview of Raman spectroscopy imaging, covering its fundamentals, a brief history and applications in the last 12 years (2006-2018).

\section{History: from the Discovery of the Pheno- menon to Spectroscopy Image Spectroscopy}

The theoretical prediction of Raman scattering was made in 1923 by Smekal ${ }^{7}$ and experimentally proven in 1928 by researchers from two independent groups: Raman and Krishnan ${ }^{8}$ in India and Landsberg and Mandelstam ${ }^{9}$ in Russia. However, the discovery of this phenomenon was attributed only to the first group, and the effect was not only named as a tribute to Raman but he also was awarded a Nobel Prize in Physics in the year 1930 in recognition of this achievement. However, the first commercial Raman spectrometer was only built in 1953, after the development of the monochromator, using mercury at a wavelength of $435.8 \mathrm{~nm}$ as radiation source. Thus, before the 1960 's, it was possible to identify more than 40,000 compounds using this configuration. However, the source used made the application difficult, especially for photosensitive and colored samples that intensely absorbed this radiation..$^{10}$ Thus, the invention of the laser in 1960 and its use as a light source in Raman spectrometers, being introduced by Brazilian physicist Sérgio Porto and Wood ${ }^{11}$ in 1962, presented another great advance in this type of spectroscopy, allowing its application in a faster and more reproducible manner. ${ }^{11}$
As previously mentioned, the first suggestion of coupling a microscope to a Raman spectrometer was in 1966. However, this equipment was developed only in 1974 by Delhaye and Dhamelincourt. ${ }^{2}$ This new technique allowed the use of Raman spectroscopy for surface mapping. ${ }^{3}$ Another important step was the use of chargecoupled device (CCD) detectors from 1987. ${ }^{12}$ Since Raman scattering presents an inherently weak signal, this problem has been solved by using CCD detectors, improving Raman sensitivity and further extending the range of applications. Starting in the 1950's, several variations of Raman spectroscopy were also developed, such as coherent anti-Stokes Raman spectroscopy (CARS), surface enhanced Raman spectroscopy (SERS), resonance Raman spectroscopy (RRS), tip enhanced Raman spectroscopy (TERS) and stimulated Raman spectroscopy (SRS) which brought significant improvements in several aspects (speed and/or resolution) compared to spontaneous Raman. The main events of the development of Raman spectroscopy imaging from 1920 to 2014 are shown in Figure S1 (in the Supplementary Information (SI) section).

Raman spectroscopy presents many of the advantages of vibrational techniques, such as little or no sample preparation and direct and non-destructive analysis, considered important mainly in cases of the need for sample preservation, such as with works of arts, artefacts or forensic analysis. ${ }^{13,14}$ Another advantage is the possibility of performing analyses in the presence of water since it does not interfere with the spectra, unlike infrared spectra. ${ }^{15}$ Another important characteristic is that both organic ${ }^{16,17}$ and inorganic compounds ${ }^{18,19}$ present Raman signals, ${ }^{20}$ which allow both types to be analyzed using this technique.

As mentioned in the Introduction section, this technique combines Raman spectroscopy with a digital image, so that, in addition to all the advantages mentioned above, the spatial information of the sample is aggregated. ${ }^{21}$ This allows the visualization of the distribution of compounds in the sample, so that it is possible to determine the homogeneity of the distribution, ${ }^{22,23}$ understanding the physical properties of the materials, ${ }^{24}$ and permitting the visualization of biological processes, ${ }^{25}$ among others.

Another advantage refers to the possibility of detecting smaller amounts of analyte compared to point spectroscopy. In point spectroscopy, one spectrum per sample, which represents the average sampled volume (often called bulk in the literature) is obtained. Thus, in many cases, compounds in low concentrations are not detected. However, since in imaging spectroscopy an area of the sample is analyzed, even if the analyte is absent in several pixels, it may have a high concentration in other pixels, which allows it to be detected even at low concentrations. ${ }^{26}$ 
The main disadvantages of Raman imaging are spatial resolution, sample components with intrinsically low Raman signals, fluorescence, subsampling, spherical aberration/refraction and problems in signal focus or force. Spatial resolution is limited by diffraction, which depends on spot size, usually in the micrometer range. The lack of Raman signal or fluorescence depends on the type of sample, where the former is a consequence of polarizability of the molecule while the latter occurs when the laser has enough energy to promote an electron to an excited electronic orbital (described in more detail in the Theoretical Fundamentals section). The problem of subsampling occurs when the mapped area is too small and is thus, unrepresentative of the sample. A more detailed description of each disadvantage can be found in a review made by Smith et al. ${ }^{27}$

\section{Theoretical Fundamentals}

\subsection{Raman scattering}

Vibrational spectroscopy is related to transitions between vibrational energy levels of the molecule. ${ }^{10}$ It is based on one of three phenomena that occur when electromagnetic radiation interacts with a molecule: absorption, transmission and scattering. ${ }^{28}$ While the first two phenomena are related to middle infrared spectra (IR), the latter is the one responsible for Raman spectra.

The intensities of the bands in the infrared and Raman spectra depend on how effective the transfer of energy to the molecule is, and this mechanism differs between these two techniques. ${ }^{28}$ The condition for infrared absorption is that there is a variation in electric dipole moment of the molecule. This implies that polar bonds tend to present greater signals than nonpolar bonds. However, in Raman scattering, the activity depends on the variation of the induced dipole moment. Thus, the opposite to infrared occurs: nonpolar bonds present greater Raman signals than polar bonds. ${ }^{10,28}$ Due to this aspect, the two techniques, even while presenting signals in the same spectral range, should not be seen as duplicates of each other, but as complementary techniques. Regarding the imaging techniques using infrared and Raman, in infrared, the analysis can be much faster, while in Raman it is possible to obtain more detailed images, and lower concentrations can be more easily detected. ${ }^{29}$

As mentioned in the Introduction section, the current energy sources of Raman spectroscopy are lasers. Monochromatic light is focused on the sample, the incident photons go into virtual state and then the scattered photons are measured (Figure 2). The so-called virtual state is not real and does not refer to any pre-existing electronic or vibrational state in molecule, but is created at the time of laser incidence. ${ }^{30}$

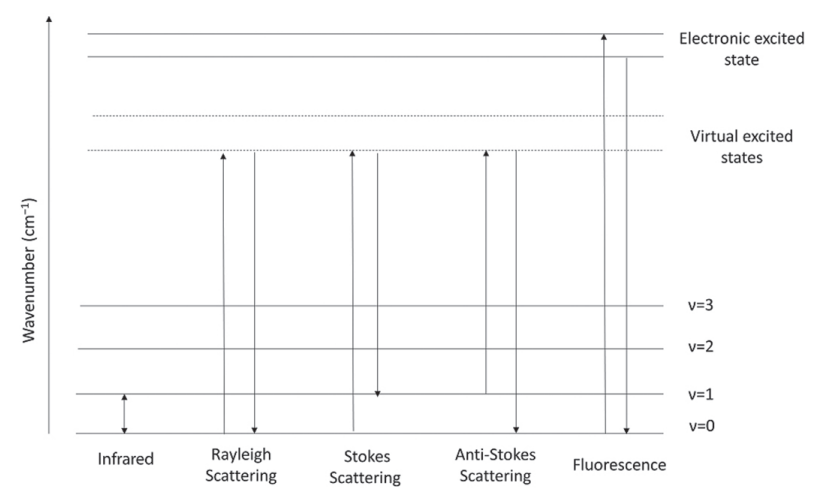

Figure 2. Schematic illustration of absorption in middle infrared (IR), Rayleigh scattering, Stokes scattering, anti-Stokes scattering and fluorescence. The upward arrows represent the excitation while downward arrows indicate the emission.

The scattering of the radiation can be subdivided into Rayleight, Stokes and anti-Stokes..$^{21}$ The most common is the Rayleigh scattering, in which no exchange of energy is involved. In other words, photons will be scattered with very small energy differences and, thus, return to the fundamental state $(v=0)$ (Figure 2). Since there is practically no difference in energy between the incident and scattered photon, Rayleigh scattering is called elastic scattering. When photons interact with the molecule and cause a polarization, there is a difference in energy between the incident and the scattered photon, and therefore it is called inelastic scattering. If the energy is transferred from the photon to the molecule, the scattered photon has a lower energy and a longer wavelength and is called Stokes scattering. If the opposite occurs, it is called anti-Stokes scattering (Figure 2). This energy difference of inelastic scattering is equal to the energy difference between the excited $(v=1)$ and fundamental vibrational states in a similar way to that found in the mid infrared. However, in infrared, there is the direct excitation of molecule in the fundamental state $(v=0)$ to the first vibrational state $(v=1)$ by a photon that has exactly the same energy difference. ${ }^{10,28}$ Light scattering is a rare phenomenon and most of it occurs in an elastic way (Rayleigh scattering). Most of the measured Raman scattering is Stokes since the molecules at room temperature are mainly in the fundamental state. ${ }^{27}$ Only a small portion of scattered light refers to the anti-Stokes phenomenon.

Considering that the Raman signal strength is proportional to the fourth power of the laser wavelength, the initial impression would be that the use of more energetic 
lasers would provide better perfomance. However this is not always favorable since instead of the photon reaching the virtual state, it could acquire enough energy to reach the electronic excited state generating a fluorescence spectrum (Figure 2) whose signal is more intense and obscures the Raman scattering. ${ }^{31}$

Therefore, aiming at improving the technique, mainly in relation to sensibility, analysis time and spatial resolution, new technologies based on the Raman scattering phenomenon were developed: SERS, CARS, TERS, SRS and RRS.

\subsection{Surface enhanced Raman spectroscopy (SERS) and tip-enhanced Raman spectroscopy (TERS)}

SERS has received much attention in recent years due to its better sensibility when compared to spontaneous Raman scattering. While the latter has a sensibility around $10^{-3} \mathrm{~mol} \mathrm{~L}^{-1}$, SERS can reach single-molecule detection, i.e., the detection of $1 / \mathrm{N}_{\mathrm{A}}\left(1.66 \times 10^{-24}\right) \mathrm{mol}$ (where $\mathrm{N}_{\mathrm{A}}$ is Avogadro's number) or 1.66 yocto mol of a compound. ${ }^{32,33}$ It is based on the plasmonic effect in which the molecules are adsorbed on a metallic surface (usually nanoparticles) resulting in high intensities of scattering, reaching improvement factors of $10^{14}$ to $10^{15}$ in relation to spontaneous scattering. ${ }^{34,35}$ The signal improvement is due to two effects: chemical improvement and the magnetic field effect, the latter being dominant. This technique has been studied in several biological applications, including in vivo studies. Comprehensive reviews on SERS can be found in the literature, among them those of Guerrini and Graham, ${ }^{36}$ McNay et al. ${ }^{37}$ Pieczonka and Aroca ${ }^{32}$ and Schlücker. ${ }^{38}$

TERS can be considered as a combination of the chemical sensitivity of SERS allied with the high spatial resolution of a scanning probe microscopy. This technique allows chemical images to be obtained below the limit of light diffraction. ${ }^{39}$ Like SERS, it also achieves singlemolecule sensitivity. However, it has a better spatial resolution: while SERS has a resolution in the range of $\mu \mathrm{m}$, TERS achieves resolutions in the range of nanometers. ${ }^{33}$ In this technique, a metallized tip is placed at a very short distance from the sample $(<10 \mathrm{~nm})$. Frequently, tips of atomic force microscopy (AFM) are coated with gold or silver to achieve signal improvement (similar to a SERS nanoparticles substrate).$^{40}$ Due to its high spatial resolution, it has been used in studies of materials and cells. In addition, due to the possibilities of many different applications, several reviews on TERS have been published in recent years, those made by Pettinger et al.,$^{40}$ Sharma et $a l .{ }^{41}$ and Kumar et al..$^{42}$ can be highlighted.
3.3. Resonance Raman spectroscopy (RRS), coherent anti-Stokes Raman spectroscopy (CARS) and stimulated Raman spectroscopy (SRS)

RRS aims at improving the detectitvity and selectivity of Raman scattering. As mentioned before, the Raman signal is inherently weak, but can be improved by several orders of magnitude when the wavelenght of the laser is close to the electronic absorption band that involves the resonance phenomenon. The smaller the difference between the laser frequency and the electronic transition, the stronger is the signal. However, there is a higher risk of fluorescence, and only vibrations coupled with chromophore groups are intensified. One way to prevent/ reduce fluorescence is using a laser in the ultraviolet range. ${ }^{43}$ A review of this technique was published in 2008 by Efremov et al., ${ }^{43}$ containing a brief history and the main applications.

Although the cited techniques present better spatial resolution and/or sensibility, they are usually slow. The issue of speed becomes crucial especially in in vivo studies. In this sense, two nonlinear Raman techniques (i.e., when the signal intensity does not depende linearly on excitation intensity) can be highlighted: CARS and SRS. Both are considered ultra-fast (around $10^{-15}$ to $10^{-6} \mathrm{~s}$ per spectrum) and present good sensibility $\left(10^{-6} \mathrm{~mol} \mathrm{~L}^{-1}\right){ }^{33}$ Since at room temperature most electrons are at the fundamental energy level, anti-Stokes scattering is very weak in relation to all the other Raman scattering phenomena. Aiming at increasing this signal, in the CARS technique, the first step consists in exciting the electrons to the first vibrational level. For this purpose, two pulses of lasers focused on the same point are used: the first promotes the electrons to vibrational level 1 and the second pulse takes the electrons to a second virtual state of energy, which later loses this energy, returning to the fundamental state $(v=0)$. Unlike CARS, in SRS, there is a dissipative process in which energy is transferred from the input photons to the molecular vibrations. Two pulsed lasers are also used, but the frequency is chosen so that it is equal to the intrinsic vibrational resonance of the sample. ${ }^{33}$

Even though these two phenomena are nonlinear, there are important differences between the two techniques. These differences imply that the quantitative interpretation of SRS is easier than CARS (due to the linear dependence on concentration), and CARS signals are easily detected when the resonant signal is much stronger than the nonresonant background signal. The practical consequence for this is that CARS is limited to $\mathrm{CH}-$ stretching vibrations, while SRS can be applied in the fingerprint region (600 to $1800 \mathrm{~cm}^{-1}$ ). Nevertheless, due to the ease of detection of CARS signals, this is used more often than SRS. ${ }^{44}$ 


\section{Forms of Data Acquisition - Point Mapping, Line Mapping and Imaging}

Two main techniques of acquiring the data cube are available: mapping and imaging. Mapping consists in obtaining the spectra from the movement of the sample in relation to the laser, while in the imaging technique, the spectra are obtained without this movement. Despite this differentiation, these two terms are currently used as synonyms in the literature. Figure 3 presents the different modes of data acquisition, where in red the amount of pixels/wavelengths that can be obtained at each reading is highlighted.

Within the mapping technique, there is point-to-point mapping or linear scan mapping. The first is also called point mapping, point-by-point mapping or raster mapping, while the latter is called line-scanning mapping. In point-to-point mapping (Figure 3a), the laser is focused on a point in the sample and a spectrum is acquired at this point/pixel, then the sample is moved and a new spectrum is acquired in the same region, and so on. In this case, the cube presents high spectral resolution, but low definition images. This is the most widely used method for obtaining the data and it provides more detailed images, although it is the most time-consuming. Linear scan mapping (Figure 3b) acquires the spectra of a line of pixels: the laser is defocused to cover all pixels in one direction, so that the data acquisition is faster than in the point-by-point method. However, since laser illumination is uneven, the spatial resolution of the image is not as good as in the first method. ${ }^{21}$

Imaging techniques are also called global imaging or wide-field Raman imaging. In this case, the laser, as in linear scanning, is also defocused, but now it illuminates the entire sample, allowing the acquisition of spectra of the entire region simultaneously (Figure $3 \mathrm{c}$ ). The vast majority of imaging techniques acquire the spectra in slices of the cube, i.e., one wavelength of the spectrum at a time. This makes it a faster method when the spectral range to be analyzed is less than the number of points in point-by-point mapping or than the square root of the number of points in linear mapping. The development of a method using a fiber-optic arrangement, called Fiber Array Spectral Translation (FAST), allows all spatial and spectral information to be obtained simultaneously, making data acquisition much faster. ${ }^{21}$ One of the problems encountered in linear mapping and imaging methods is that the laser is defocused. Since the Raman signal is low, this can significantly increase the possibility of fluorescence interference. Comprehensive reviews on Raman imaging spectroscopy have been presented, highlighting those of Stewart et al. ${ }^{21}$ and Opilik et al. ${ }^{45}$

\section{Treatment of Data}

\subsection{Data preprocessing}

Before using the Raman spectra for qualitative/ quantitative purposes either by univariate or multivariate methods, a very important step is data preprocessing. The aim of this step is to eliminate disturbances that occur during the analysis or to limit the effect of undesired phenomena associated with the sampling arrangement and/or sample emission. In Raman spectra there are five main spectral artifacts to be corrected: baseline, noise, differences in scale, spikes and dead pixels..$^{27,46}$

Due to the high sensitivity of the CCD, one of the problems encountered is the detection of cosmic rays, which contaminate the Raman spectra with random, positive, unidirectional and relatively fine signals. As shown in Figure S2a (in the SI section), the presence of spikes alters the Raman spectrum, limiting the performance of multivariate methods due to the introduction of problems such as distortions, false pure variables and wrong identification of components in classifications. ${ }^{47}$ Therefore, it is extremely important to eliminate this signal before the development of models.

Noise in the data is usually corrected using the Savitzky-Golay smoothing algorithm, which is based on the application of a polynomial filter to the spectral data. It is necessary to choose two parameters, the width of the filter window and the order of the polynomial to be used. ${ }^{46}$ Larger windows and lower grade polynomials provide greater (a)

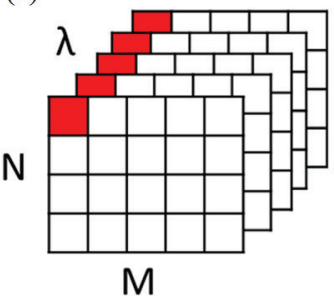

(b)

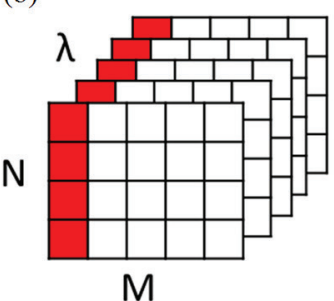

(c)

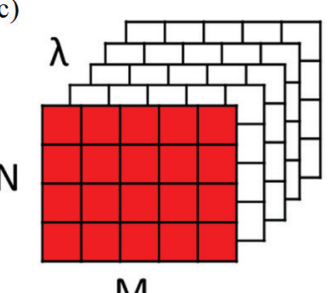

Figure 3. (a) Point mapping, (b) line-scanning mapping and (c) imaging. 
smoothness; however, distortion or even disappearance of peaks may occur if the window is too wide and/or it uses a low grade polynomial (Figure S2b in the SI section).

Another problem that arises in the spectra is the shift of the baseline, which may occur due to scattering or fluorescence and is generally not related to the chemical composition of the sample. One of the forms of correction is the use of derivatives ${ }^{27}$ or the application of polynomials such as asymmetric least squares ${ }^{48}$ or weighted least squares (Figures S2c and S2d in the SI section). On the other hand, problems of differences in scale are usually caused by differences in focus due to a non-homogeneous surface and can be corrected using normalization methods, with emphasis on the unit vector (the length of the vector/spectra is equal to one). Finally, dead pixels are usually due to anomalies in detectors, which can have zero or lost values in pixels. Like spikes, they distort values, and therefore it is important to identify and eliminate them at this stage. The most common form of correction is to replace these pixels with the median or the average of the neighboring values. ${ }^{49}$

Sacré et $a l .{ }^{46}$ presented a review on data processing in chemical images obtained using vibrational spectroscopy, while Vidal and Amigo ${ }^{49}$ showed some problems encountered in the treatment of spectral images, as well as ways to circumvent them.

\section{Data Analysis}

Regardless of the form of acquisition of the cubes, the form of data treatment is the same and can be divided into two categories: univariate and the multivariate. In the first one, only one value is chosen (e. g., area of a band or height of a peak) for the data analysis; in other words, it would be like choosing one of the slices of the hypercube to represent the analyte of interest. In the case of the multivariate form, the full Raman spectrum is used in the analysis.

A bibliographic research was carried out on Raman spectroscopy publications from 2006 to 2017, using as keywords: "Raman imaging", "Raman mapping" and "Raman hyperspectral" in the title and/or subject areas of the Capes/MEC Periodic Portal (periodicos.capes.gov. br) (where Capes is Coordenação de Aperfeiçoamento de Pessoal de Nível Superior and MEC is Ministry of Education, both from Brazil) and in topic and/or title in the Web of Science (webofknowledge.com), obtaining a total of 1,468 published articles. Univariate methods were the most used $(76 \%)$, while multivariate methods were used in only $21 \%$ of the publications. The remaining percentage $(3 \%)$ is of articles comparing these two forms of treatment. This percentage has not changed much over time (Figure 4); the percentage of articles using the univariate method usually varies between 70 and $80 \%$, and that of the multivariate method between 15 and $25 \%$ of all articles. The univariate method is the simplest and most direct method that can be used for data analysis, but it may present problems when dealing with more complex matrices or greater signal overlap. In these cases, use of multivariate data is indicated and, although more complex, more complete information is extracted.

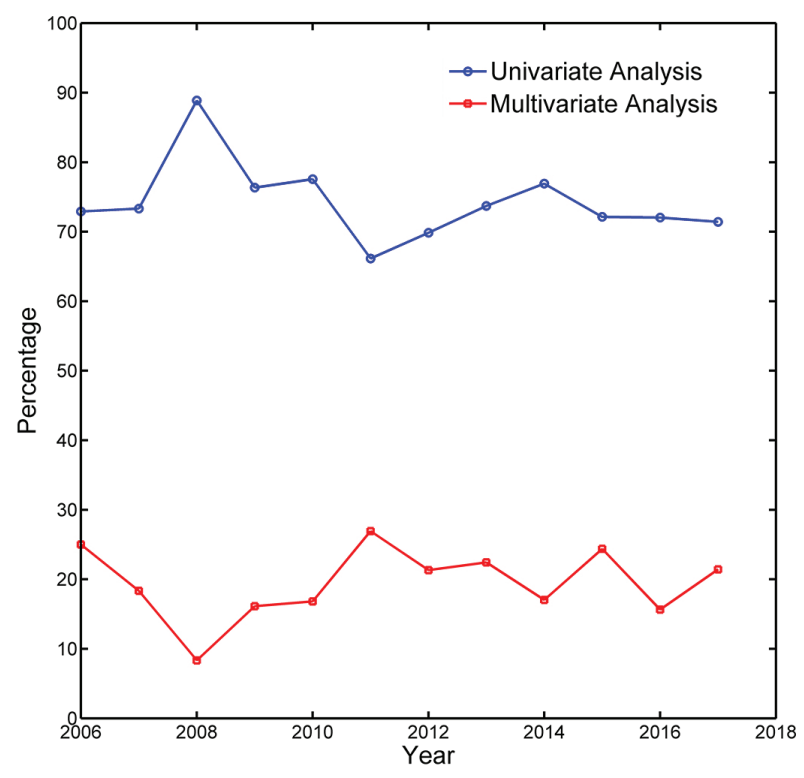

Figure 4. Variation of Raman data treatment over the time (2006-2017).

\subsection{Univariate methods}

Four types of univariate methods are normally used: area under a peak, height of a peak, ratio between the height of two peaks or use of a function for signal separation. The first two are used when the peak referring to the analyte of interest is well resolved and without overlapping peaks from other components of the mixture. The other two cases are used when there is an overlap of bands and/or when another component of the sample also presents a signal in that region. The most used is the height of a peak, that is, the intensity of the peak of the analyte of interest (Figure 5a). The integral of a peak of interest, that is, the area, is the second most used method (Figure 5b).

The overlapping of peaks in infrared and Raman spectra is very common, which makes it difficult or even impossible to use the area or intensity of the peaks. The univariate alternatives are the ratio between the heights of two peaks (Figure 5c) and curve adjustment by a function (Figure 5d). The ratio between two peaks is mainly used in analyses of graphene samples and plant cell walls, with emphasis on the first. Graphene has two main bands in the Raman spectrum, called $\mathrm{G}$ and $\mathrm{D}$, whose intensity ratio, 
(a)

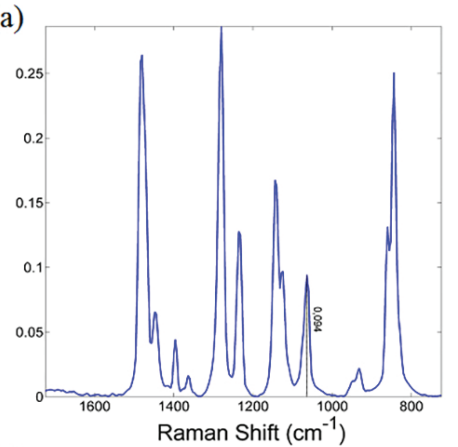

(c)

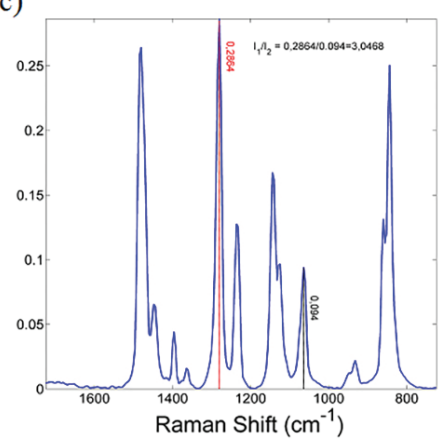

(b)

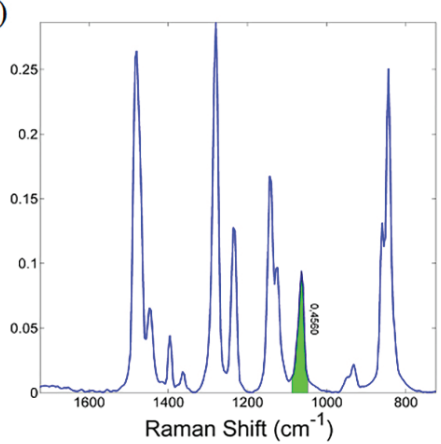

(d)

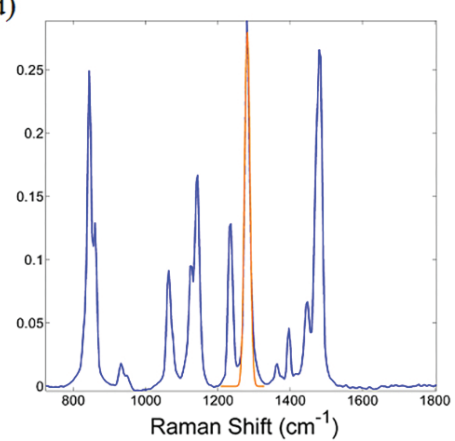

Figure 5. Different forms of univariate analysis: (a) intensity, (b) area, (c) ratio between intensities and (d) gaussian function fit.

as well as the position and shape of the peaks, provides an idea of the degree of disorder of the material. ${ }^{50}$ The adjustment of a function is a technique that reveals the individual vibrational bands in the spectra of a given region. The main functions are Lorentzian, Gaussian and Voigt. This is because the individual spectral bands in infrared and Raman present the lorentzian form, while instrumental or sample effects usually generate Gaussian curves. ${ }^{51}$ The Voigt function consists of a mixture of the two previous functions. After application of the adjustment, the area of the band is calculated and used for the construction of the chemical maps.

\subsection{Multivariate methods}

The main multivariate methods used for the treatment of Raman imaging data are principal component analysis (PCA), vertex component analysis (VCA), band-target entropy minimization (BTEM), classical least squares (CLS), multivariate curve resolution (MCR), partial least squares (PLS) and partial least squares- discriminant analysis (PLS-DA).

All the different methods begin with the process of unfolding the cube. In the unfolding, the pixels are placed one below the other (that is, in the rows), while the columns represent the variables (Figure 6a). After the unfolding, a chemometric method is applied, generating values that represent each pixel (for example, the PCA scores). These values are refolded, thus obtaining the concentration maps. The exceptions are the methods that require calibration (PLS and PLS-DA, for example). In these cases, the mean spectrum of each data cube is calculated (Figure 6b), a calibration model is constructed using these mean spectra and the values of the property of interest (or class in the case of PLS-DA). This model is applied to the unfolded matrices, and the concentration vectors obtained are refolded for the construction of the chemical maps. One of the main disadvantages of calibration methods in relation to other methods is the number of samples required, usually a much larger number than for the curve resolution methods.

PCA is one of the most important methods in chemometrics and widely used in spectral imaging treatments. It is an exploratory method used to reduce the amount of data based on the correlation between variables. ${ }^{52}$ Using PCA, hundreds or thousands of variables are reduced to a few, called principal components (PC). The decomposition of the data can be represented by equation 1:

$X=\mathbf{T P}^{\mathrm{T}}+\mathbf{E}$

where $\mathbf{X}$ is the initial data matrix $(\mathrm{N} \times \mathbf{M})$, $\mathbf{T}$ is the score matrix $(\mathrm{N} \times \mathrm{A}), \mathbf{P}$ is the loading matrix $(\mathrm{M} \times \mathrm{A}), \mathbf{E}$ is the residual matrix $(\mathrm{N} \times \mathrm{A}), \mathrm{N}$ is the number of samples, $\mathrm{M}$ the number of variables and $\mathrm{A}$ is the number of PC. The direction of the main components is determined in order to capture the greatest possible variance of the 
(a)

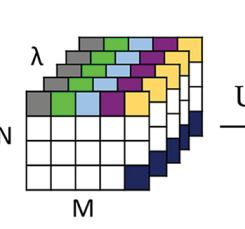

Data Cube
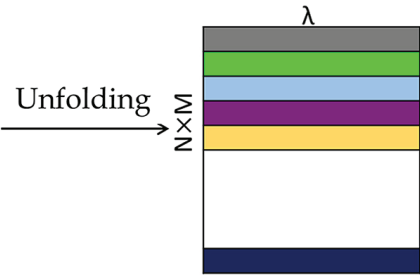

Unfolded

Matrix
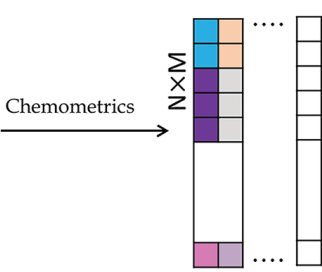

A Scores vector

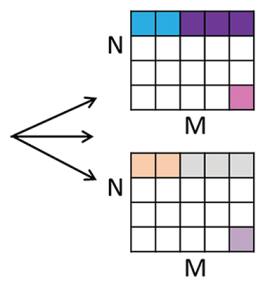

$\mathrm{N}$

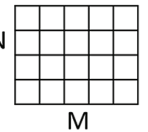

A chemical images

(b)
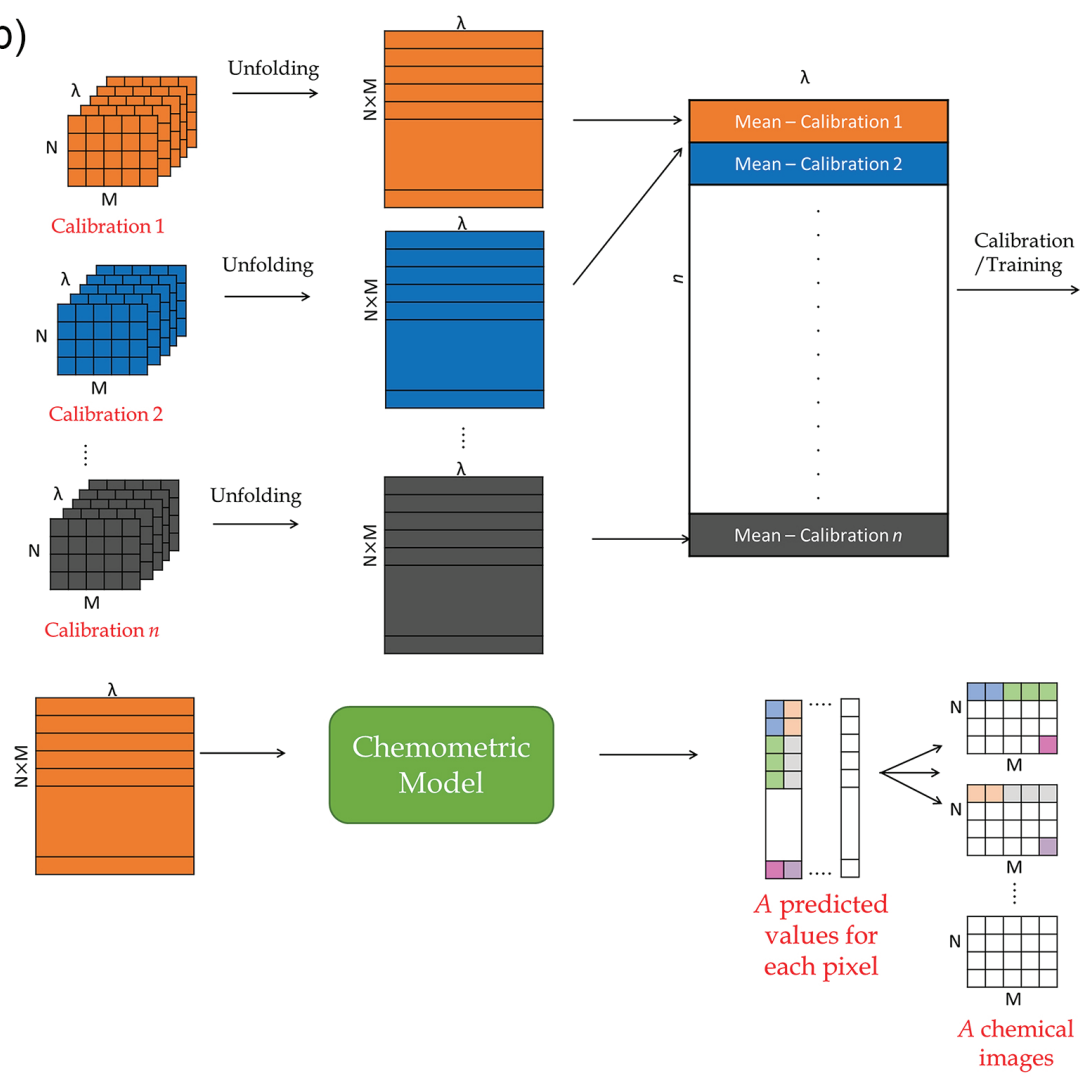

Figure 6. Generation of chemical images (a) without and (b) with calibration methods.

data. ${ }^{53,54}$ The scores are the values of the objects in reduced dimensionality, while the importance of the original variables for the PC is shown in the loadings. Residuals are the part of the $\mathbf{X}$ matrix not described by PC and are usually just noise. In the literature, there are several tutorials and/ or reviews on PCA, with a highlight to the one published by Bro and Smilde ${ }^{53}$ in 2014.

VCA is a relatively new unsupervised method used to find the profiles of pure compounds in a mixture. ${ }^{55}$ This algorithm estimates the number of reference substances (called endmembers), the spectral signature and their fraction in the mixture. It assumes that there are pure pixels and projects the data in an orthogonal direction to the subspace determined by the endmembers. VCA can be represented by equation 2 :

$\overrightarrow{\mathrm{r}}=\mathbf{x}+\mathbf{n}=\mathbf{M} \gamma \vec{\alpha}+\mathbf{n}$

where $\vec{r}$ is a vector with $L$ bands, $\mathbf{M}$ is the mixing matrix, $\gamma \vec{\alpha}$ is a scale factor that models the variability due to the topography of the analyzed surface, $\vec{\alpha}$ is a vector containing the fraction of each endmember and $n$ is the residue. The algorithm is described in the work of Nascimento and Dias, ${ }^{55}$ published in 2005. 
BTEM is also a recent method, developed in 2002 by Chew et al. ${ }^{56}$ It was initially used for organometallic analysis in IR spectra, but over time, it has been applied to other areas, including Raman imaging data. ${ }^{56}$ It is used to extract spectra of pure components and their spatial distributions without a priori information about the constituents of the sample. ${ }^{56}$ BTEM searches for an individual characteristic from the decomposition of the space vector of the observations/samples and subsequently reconstructs the corresponding spectrum of the pure component. Since entropy is a measure of system disorder, low values imply an organized and simple system. Since pure spectra are supposed to be the simplest fundamental patterns, minimizing entropy seems adequate to find them..$^{57}$

Like PCA, classical least squares is one of the most used methods in imaging processing. Some articles use CLS (classical least squares) or DCLS (direct classical least squares) to refer to this chemometric method. This method is based on equation 3 :

$\mathbf{X}=\mathbf{C S}^{\mathbf{T}}+\mathbf{E}$

where $\mathbf{C}(\mathrm{p} \times \mathrm{k})$ is the matrix containing the concentration profiles of k compounds for p samples, $\mathbf{S}(\mathrm{k} \times \lambda)$ are the spectral profiles with $\lambda$ variables of $\mathrm{p}$ compounds and $\mathbf{E}$ $(\mathrm{k} \times \lambda)$ is the residual matrix. This method is relatively simple, and only requires matrix $\mathbf{S}$ for the calculation of $\mathbf{C}$ (or vice versa), and is often incorporated into commercial programmes; an example is Renishaw's WiRE ${ }^{\mathrm{TM}}$ program. However, it has the disadvantage of requiring all the $\mathbf{S}$ spectra (or $\mathbf{C}$ concentrations) to be known profiles and cannot deal with interactions between compounds/spectral changes due to sources other than concentration. ${ }^{27}$

MCR refers to a group of methods used to solve the problem of mixture analysis, and the most important member of this group is multivariate curve resolution with alternating least squares (MCR-ALS). The MCR-ALS equation is similar to that of CLS (equation 3), however, as opposed to the previous one, this method allows the analysis even in the presence of interactions among the components of the mixture. This occurs due to its iterative resolution: in a first step, the concentration profiles are calculated using equation 3. Then, using these concentration profiles $\left(\mathbf{C}_{1}\right)$, new spectral profiles are calculated $\left(\mathbf{S}_{1}\right)$. Using $\mathbf{S}_{1}, \mathbf{C}_{2}$ is calculated and so on, until a certain number of iterations or until the differences between the values of one interaction and another is less than a certain limit. ${ }^{58}$ de Juan et al..$^{58}$ published a review of MCR, describing not only the methods, but also the use of constraints and other ways to improve resolution.
PLS is one of the most important methods of multivariate regression and can be readly applied to image analysis. It is a method of inverse calibration, in which a matrix of $\mathbf{Y}$ dependent variables (for example, concentrations) is correlated to a matrix of $\mathbf{X}$ independent variables (in this case, the mean of the spectra of the analyzed area) through a vector of coefficients $\vec{b}$ (equation 4): $:^{59}$

$\mathbf{Y}=\mathbf{X} \overrightarrow{\mathbf{b}}+\mathbf{E}$

In the case of the partial least squares, discriminant analysis (PLS-DA), the only difference refers to the matrix $\mathbf{Y}$, instead of values referring to a property of interest, this vector contains whole values referring to a certain class (e.g., healthy cells/sick cells). Geladi and Kowalski ${ }^{59}$ published a well-known tutorial on PLS in 1986, while a tutorial review on PLS-DA was presented in 2013 by Ballabio and Consonni. ${ }^{60}$

\section{Raman Spectroscopy Imaging Applications}

Raman imaging spectroscopy has seen increasing interest in several areas. A bibliographic search using the same databases mentioned in section 6 was carried out on Raman mapping/imaging spectroscopy publications from the last 12 years (2006 to January 2019). A total of 1785 published articles were found, with the number increasing over time (Figure 7). In this research, we did not consider articles that used an image spectrometer only to make point measurements, ${ }^{61,62}$ i.e., without interest in the spatial distribution/chemical image. We also found 123 review articles or perspectives on Raman spectroscopy imaging, whether referring to the technique and advances in general or its application in a given area.

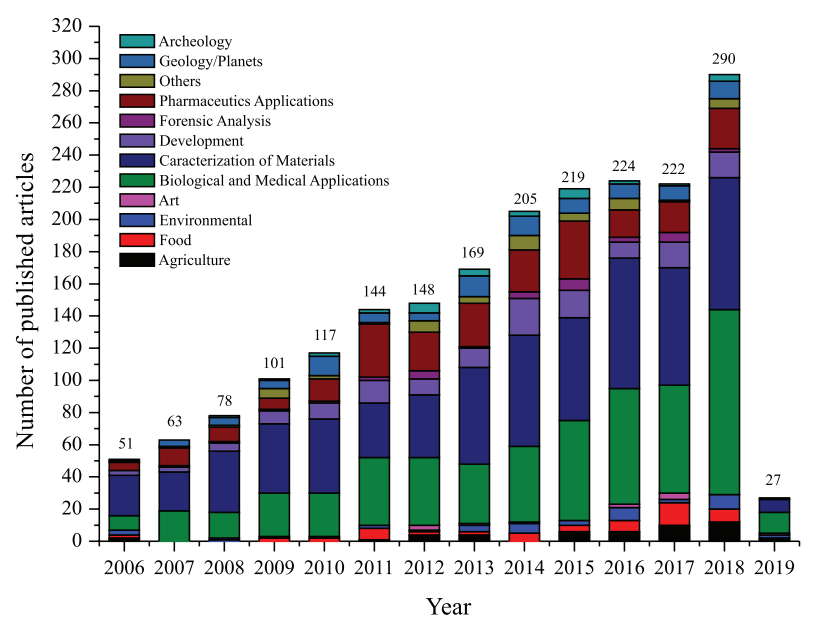

Figure 7. Number of published articles using Raman imaging from 2006 to January 2019. 
Regarding the application areas, 11 main areas were found: agriculture/biomass, environmental, biological and medical analysis, development of methods or instruments, pharmaceutical applications, geological analysis/planet and celestial bodies composition, food, arts, archaeology, material characterization, forensic analysis and archaeology. The area with the largest number of published articles in the 12 years studied is material characterization, followed by biological and drug analysis. The others represent less than $10 \%$ of the total each one. Spontaneous Raman is the most used in these studies (76\% of the total), followed by SERS, which represents $10 \%$. CARS and TERS account for $4 \%$ each. The other techniques each represent a percentage below $2 \%$ of the total. The increase in recent years in the use of techniques other than spontaneous Raman is mainly due to applications with interests in analyzing compounds at low concentrations, using shorter analysis times and/ or better image resolution, as explained in the Theoretical Fundalmentals section.

\subsection{Agriculture and biomass}

The articles on the application of Raman spectroscopy imaging in the area of agriculture and biomass during the studied period can be divided mainly into the analysis of pesticides and their effects, characterization of seeds, fertilizers and soils, studies of agricultural products, and the characterization of plant cell walls for the production of biofuel from lignocellulosic materials (Table S1 in the SI section). In the majority of cases, the Raman image was not used alone, but associated with other imaging techniques, such as SEM, TEM and AFM or with chromatographic techniques (mainly high performance liquid chromatography, HPLC) for the characterization of samples; there were few cases where Raman was used alone. .3.64 $^{2}$

There is a predominance of the use of univariate methods (71\%) and the use of spontaneous Raman (73\%) for treatment and data acquisition, respectively. SERS (19\%), resonant Raman (4\%) and SORS (4\%) techniques were found as Raman variations. The SORS method is relatively recent, developed in $2005 .{ }^{65}$ It uses spontaneous Raman, but obtains spectra from the internal part of the sample without the need to open/destroy the sample. Its potential has been exploited in seed characterization since normally these samples must be cut to obtain the infrared and Raman spectra from its internal part. With the use of SORS, this procedure was avoided and the methodology proved to be non-destructive for this type of sample. ${ }^{66}$

Biofuel production has attracted attention in recent years, but lignocellulosic materials, the most abundant in nature, present low yields for bioethanol production. One of the main reasons for this problem is the cell walls, which protect plants from external attacks. This resistance is not only a product of the quantities of compounds, but also of their spatial distribution. Therefore, Raman imaging spectroscopy has been applied, aiming at this characterization, before and after the treatments for the destruction of cell walls, trying to find which pretreatment is the most effective, or even trying to understand the mechanisms of the process.

\subsection{Foodstuff}

In the area of foodstuffs, publications were found both for the characterization of products and for studies of pesticides presence and adulteration processes (Table S2 in the SI section). Different from the other areas, there was a slightly greater predominance of multivariate methods (44\%) than univariate methods (39\%), with a significant percentage using both methods (17\%). There is a great predominance in the use of spontaneous Raman (83\%), followed by SERS (11\%) and SORS (6\%).

Wang et al. ${ }^{67}$ used SERS for the detection of bacteria in skimmed milk. ${ }^{67}$ Using this method, it was possible to construct maps and identify the presence of the species of Salmonella enterica, Escherichia coli BL21, Listeria monocytogenes 18 and Lactococcus lactis bacteria. This type of analysis is of great importance in the area of food since Salmonella is a known food pathogen and the second largest agent responsible for human gastrointestinal infections. ${ }^{68}$ Zhang et al. ${ }^{69}$ used MCR-ALS on Raman and mid-infrared images to construct maps of constituent distributions in white chocolate and milk. ${ }^{69}$ It was possible not only to identify sucrose, lactose, fat and whey, but also an unknown compound. From the maps obtained it was possible to locate the butter and whey scattered and trapped around the sugar particles. The other constituents were distributed in heterogeneously separated particulates.

Recently, Yaseen et al. ${ }^{70}$ published a review on the use of Raman images for quality control and food safety evaluation, citing recent studies involving the technique, as well as future perspectives for application in industry and research in this sector.

\subsection{Environmental}

Articles from the environmental area represent only $2 \%$ of the total number of papers, mostly focused on the effects of pollution on the environment. It is a promising area due to both the current concern with the environment and to the possibilities found, since it is an area little 
explored by Raman spectroscopy imaging to now. The form of data treatment is predominantly univariate $(71 \%)$ and the acquisition is mostly from spontaneous Raman $(93 \%)$. Table S3 (in the SI section) summarizes some of the applications found in the area.

Käppler et al. ${ }^{29}$ used IR and Raman imaging to study environmental microplastics. This material is a contaminant of aquatic ecosystems, with negative impacts on biota. The authors showed that in cases of very colorful materials, the use of both techniques can be advantageous because many fillings and pigments have Raman spectrum, but not IR. However, the use of only Raman microspectroscopy can lead to errors, especially for painted particles. The authors' results allowed distinguishing microplastics in relation to number, type and size, as well as measurement time. ${ }^{29}$ Batonneau et $a l .{ }^{71}$ analyzed urban tropospheric aerosol particles using confocal Raman allied with MCR. The results were compared to X-ray mapping and were compatible. Particles rich in lead and zinc metals mixed with mineral dust were detected in the Northeast of the city when the wind was coming from the Western sector. The authors believe that these particles were possibly suspended in the troposphere by the action of wind or mechanical disturbance of polluted soils. ${ }^{71}$

\subsection{Biological and medical applications}

This area has the second highest number of applications. It is mainly divided into two parts: studies/characterizations of cells (vegetable, animal or human) and studies of diseases, in which efforts for the diagnosis and identification of cancer cells can be highlighted (Table S4 in the SI section). The term label-free was found mainly in these applications, highlighting the use of a dye free method, such as the traditional methods of microscopy. It was the area with the greatest diversity of Raman techniques, although spontaneous Raman still represents $68 \%$ of the articles found. In many cases, the traditional method of diagnosis is histopathological analysis. This method is subjective and requires an experienced analyst. Therefore, the development of methods that are more objective in their results is highly desirable.

Studies on spatial changes and with the time of cytochemistry in colonies of human embryonic steam cells were performed by Konorov et al. ${ }^{72}$ This allowed a better understanding of the niches of these colonies and aspects of the tissue, morphologies and organ trajectories. Han et $a l .{ }^{73}$ studied the metabolism of antitumor drug 6-mercaptopurine in living cells. Using SERS, the authors could visualize the real-time distribuition of drug and its biotransformation in tumor cells. Marini et al. ${ }^{74}$ detected stretched DNA using microRaman spectroscopy and superhydrophobic substrate. This work showed that this technique can distinguish DNA molecules due to chemical conformation behaviour and can be used in several applications on bio-medical field, as interaction with toxins or proteins. Using the advantage of high spatial, sensivity and velocity of SRS, Saar et al. ${ }^{75}$ presented an in vivo video-rate molecular imaging in mice, humans of the drug penetration of topically applied drugs. In summary, Raman imaging has been used for a wide variety of matrices in biological applications: teeth, tissues, cells, monitoring of drugs in cells and their biotransformations, bacteria, biological pathways, in vivo or in vitro analysis, among others. The need for the analysis of these highly different matrices justifies the use of different variations of Raman spectroscopy (SRS, CARS, TERS, RR and SERS, for example).

Some problems are found in this type of study using the Raman image, and one of the most relevant is the analysis time. It is not uncommon for image aquisition to take several hours, which would make in vivo use difficult. Attempting to reduce time would affect image resolution, which in many cases is not desirable. An alternative is the use of other methods besides spontaneous Raman; CARS and SRS are the most used in this application since they are fast and do not need sample preparation. ${ }^{75-77}$ The use of SERS for locating particles in specific parts/organs is also an important area of research in recent years, with in vivo studies being carried out in small animals, such as mice. There is also the problem of fluorescence in this type of sample, making its application even more difficult, which is partially solved by the use of lower energy lasers, in spite of the loss in sensibility. There are several reviews on the application of Raman spectroscopy imaging in the literature for biological and medical applications. Those made by Krafft et al..$^{77}$ (use of linear and non-linear Raman imaging of cells and tissues) and by Jermyn et al. ${ }^{78}$ (advances in the area with emphasis on transference for clinical analysis, mainly in cancer treatment) should be higlighted.

\subsection{Development of equipment and methods}

Raman spectroscopy imaging is not a consolidated area yet, and there is much to be investigated, either to improve the performance of the method or the equipment. Table S5 (in the SI section) presents a summary of some applications found in this area. Duarte et al. ${ }^{79}$ for example, developed CARS microscope with Fourier transform for imaging. Using this equipment, it was possible to obtain an image of the entire region at the same time, decreasing the damage to the sample. ${ }^{79}$ Modifications in the TERS 
tips are also found, aiming at improving the performance of the method, ${ }^{80,81}$ the techniques to improve data (e.g., removal of spikes or improving signal to noise ratio), ${ }^{82,83}$ the development of SORS for linear mapping, ${ }^{84}$ and others. It is possible to find articles that try to develop new equipment or do modifications for new applications and/or performance improvements, as well as new modes of data processing. Most of these articles on equipment are not found in the area of chemistry, but in articles focused on the areas of physics and engineering, dealing with the optics, such as Microelectronic Engineering, Applied Physics Letters, Optics Communications and Optics Letters, among others. The variants SRS, CARS, TERS and SERS, being more recent techniques, are the ones that appear more often in this area, with more prominence for the non-linear techniques CARS and SRS.

\subsection{Geology analyses and composition of planets and celestial bodies}

Raman analyses in these fields usually occur along with other imaging techniques, mainly SEM/EDS. The objectives of the studies are diverse, from the characterization of materials to the impact of living organisms on a given environment (Table S6 in the SI section). A review on the use of Raman spectroscopy in planetary materials was published in 2017 by Chou and Wang. ${ }^{85}$

An interesting study on the application of this technique to terrestrial and extraterrestrial materials was conducted by Wang et al. ${ }^{86}$ in five different extraterrestrial materials. From the distribution of the compounds obtained in the maps, it was possible to describe which processes, such as impact, each sample went through or with which materials it came into contact. The work developed by Frosch et al. ${ }^{87}$ analyzed meteorites of Mars using different sources of excitation and resonant Raman. These meteorites have fluorescent compounds, which may interfere with the analysis. The use of less energetic lasers may not be the best solution for this case because rare earth impurities are present and present levels of electronic excitation at relatively low energies. Thus, the authors highlight the use of excitation in the ultraviolet instead of the region of the visible or near infrared. Using an ultraviolet source, the problem of fluorescence is avoided, Raman signals are more intense and selectivity is improved. ${ }^{87}$

\subsection{Arts, archeology and paleontology}

These three areas will be discussed together because they have the same characteristics and in many cases are interrelated. Univariate methods prevail over multivariate methods. This may happen because the main focus in these analyses is in inorganic rather than organic compounds; thus, it is easier to find characteristic bands of the compounds, and build a map based only on them. Moreover, usually Raman image spectroscopy is not the focus of research, but is used in conjunction with other imaging techniques (such as SEM/EDS). These studies with Raman are still in the early stages, and in many cases the data analysis is performed by the equipment's own software. An example of this can be found in the work of García-Florentino et al. ${ }^{88}$ in the Punta Begoña gallery built in 1918. They analyzed part of the wall mortars to find the effect of time on the different types of materials. It was also shown that dolomite was found in the mapping, but not in the point Raman analysis. ${ }^{88}$

An interesting technique for use in works of art is SORS, briefly described in the agriculture/biomass application section. As the sample is preserved, but internal information is obtained from the sample, its application in artwork is promising. An example of this use in cultural heritage can be seen in the work of Botteon et al. ${ }^{89}$ in which they identified and created maps of layers of various pigments and raw materials hidden in the paintings. ${ }^{89}$

\subsection{Material characterization}

This was the area with the highest number of applications (33\%) and presents a predominance of univariate methods $(98 \%)$. Spontaneous Raman is also predominant, but other techniques can also be found. It was also in this area that the main applications of TERS were found. The main materials studied are polymers, catalysts and carbon compounds (mainly involving graphite and graphene). They aim at both the characterization of the materials and the effects after exposure to a given phenomenon and/or treatment. As Raman is used more for the characterization of a routine form, and not as a search tool for new forms of analysis, in most cases, it is used in conjunction with other imaging techniques, where TEM, SEM and AFM can be highlighted. Table S8 (in the SI section) presents a summary of some applications found in this area.

The aging of polymeric surfaces using IR and Raman images was performed by Eder et al. ${ }^{90}$ In this work, the complementarity of the techniques was demonstrated, with IR being more suitable for the identification of organic molecules (especially when carbonyl, hydroxyl and amine groups are present) and Raman for unsaturated compounds (such as conjugated and aromatic ones), as well as inorganic materials. An example of the use of TERS in the characterization of materials can be seen in the work of Kazemi-Zanjani et al., ${ }^{91}$ in which it was used for the 
characterization of silicon nanowires to determine if they were on their overlapping or in isolation. It was possible to find subtle spectral changes depending on the position of the nanowires due to better spatial resolution. The difference in a certain area was also demonstrated when using the TERS technique with or without the AFM tip. ${ }^{91}$

\subsection{Forensic analysis}

The book 'Introdução à Química Forense' defines forensic chemistry as: "the part of chemistry that deals with forensic investigation in the field of specialized chemistry in order to meet aspects of judicial interest". ${ }^{22}$ The main applications in this area using Raman spectroscopy imaging refer to studies of fingerprints, explosives, adulterations and fraud in documents (Table S9 in the SI section). However, many of the studies on adulteration have been included in other areas depending on the matrix analyzed (mainly drugs and food).

Fingerprint detection is important in both identification and explosive-contaminated fingerprint situations. This is due to the fact that fingerprints are unique to each individual, and therefore important forensic evidence. Using a solution of $\beta$-carotene and oil, Deng et al. ${ }^{93}$ obtained Raman spectroscopy images of fingerprints from volunteers on several types of surfaces. A good match was obtained with the real fingerprint, independent of the surface. ${ }^{93}$ Document frauds are mainly identified either by distinguishing different types of ink or by the order in which the writing was performed. Borba et al. ${ }^{94}$ used MCR-ALS to find the order of the traces of some writing and also concealment of writing. This was possible using confocal Raman because it allows the acquisition of data at different depths. ${ }^{94}$ An example using a stand-off method was provided in the detection of explosives. In this method, both the analyst and the equipment were at a certain distance from the sample, and the data could be collected without approximation. In this case, pentaerythritol tetranitrate (PETN) and ammonium nitrate $\left(\mathrm{NH}_{4} \mathrm{NO}_{3}\right)$ were analyzed at low concentrations, with the limit of detection estimated at ca. $1 \mu \mathrm{g} \mathrm{cm}^{-2}$. The stand-off analysis of explosives brings safety to the operator. ${ }^{95}$

\subsection{Pharmaceutics applications}

Raman imaging spectroscopy is an important analytical tool in the pharmaceutical industry, being used in both the development and in the quality control of drugs (Table S10 in the SI section). The spatial distribution of the compounds in a sample may be associated with their effects and with the stability of the product. Different processing methods and their effects, studies of impurities, adulterations, among others are also evaluated. Scoutaris et al. ${ }^{96}$ studied the effect of different formulation processes on the distribution of paracetamol and found that more homogeneous formulations were obtained in pre-mixed extruded formulations than those extruded by the method of hot fusion or obtained by direct compression of the drug with the excipients. ${ }^{96}$

The Raman imaging has also attracted attention in the study of different polymorphic forms of drugs since it is more sensitive than IR and near infrared (NIR) spectroscopies to the crystallinity and polymorphism of compounds. The study of polymorphism is particularly important in the pharmaceutical field as it is directly linked to formulation stability and safety. Piqueras et al. ${ }^{26}$ have developed a work to monitor the polymorphic transformations of carbamazepine, an antiepileptic approved worldwide and considered an essential drug by the World Health Organization (WHO). ${ }^{97}$ This method has allowed the detection of polymorphic forms I and III. The two forms are the most common forms of carbamazepine, with form III as the main form used in drug products, while form I is found at higher temperatures. These results can help to determine the storage conditions and optimization of the drug product manufacturing processes. ${ }^{26}$ Not only active pharmacecutical ingredients can present structural changes over the time but also the excipients, as in case of semi-solid formulations. Mitsutake et al. ${ }^{98}$ described structural changes in polyethylene-glycol/polysorbate 80 mixtures over time by different chemometric methods.

\section{Conclusions}

Raman imaging spectroscopy has proven to be a valuable tool for application in various fields of knowledge. The provision of spectral and spatial information has made it possible to relate the macroscopically observed properties to the distribution of the compounds present in the mixture. In addition, its use can be associated with other instrumental techniques, either to confirm or to extract even more information. In order to improve sensitivity, spatial resolution or analysis time, variations in Raman spectroscopy were developed. Even so, research to improve the existing instrumentation is still ongoing.

Although the use of univariate methods is predominant, multivariate methods are indicated in many cases, especially in complex matrices and/or in samples containing low concentrations of the analytes. There are some areas where Raman spectroscopy imaging is already used as a routine technique, and in others it has been studied to complement or replace other imaging techniques. It 
is possible to highlight medical research, mainly those related to cancer cell studies. The growing interest in Raman imaging spectroscopy in this area is due to the search for less subjective methods than those currently employed. In this area, new applications are found using non-linear techniques, such as CARS and SRS, due to the shorter analysis time, which is particularly important for the development of in vivo studies. Studies in material characterization highlight the potential of TERS, which allows obtaining a resolution of nanometers, below the limit of light diffraction, in which SERS and RRS have been demonstrated to be important to improve the sensibility of the technique.

\section{Supplementary Information}

Supplementary information is available free of charge at http://jbcs.sbq.org.br as PDF file.

\section{Acknowledgments}

The authors thank CNPq (H. M. scholarship, 142441/2015-5), CAPES (finantial code 001) and INCT-Bioanalitica (FAPESP/INCT 14/50867-3; CNPq/ INCT 465389/2014-7) for financial support, and Carol Hollingworth Collins for the careful review of English.

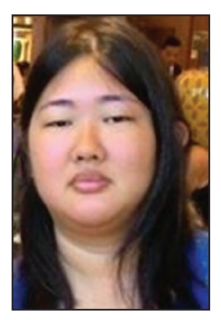

Hery Mitsutake has earned her bachelor's in industrial chemistry and her master's degree in Chemistry at the Institute of Chemistry, Federal University of Uberlândia (UFU), Brazil. In 2019, she received her PhD's degree in Science at the Institute of Chemistry, State University of Campinas (Unicamp, Brazil). She is a post-doctoral researcher at Institute of Biology (Unicamp, Brazil) since March 2019. Her current research focuses on evaluation of the miscibility of lipid excipients for pharmaceutical formulations using Raman imaging and chemometric methods.

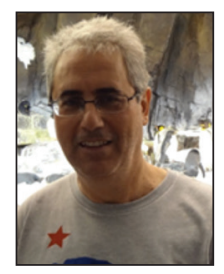

Ronei Jesus Poppi is full Professor at Institute of Chemistry, State University of Campinas (Unicamp, Brazil). He is head of the Laboratory of Chemometrics in Analytical Chemistry and member of the National Institute of Science and Technology for Bioanalytics. He has been working for several years on the development and application of chemometric tools, such as pattern recognition, multivariate calibration, multivariate curve resolution and learning machine for data treatment of instrumental analytical techniques, mainly near-infrared spectroscopy and Raman spectroscopy.

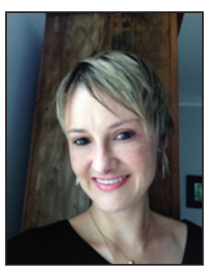

Márcia Cristina Breitkreitz was a senior researcher in Chemometrics (2006-2007) and coordinator of Research and Development (2007-2009) at the International Institute of Pharmaceutical Research (IIPF, Brazil). She received her PhD in Chemistry from the State University of Campinas (Unicamp, Brazil) in 2013. She is a Professor of Analytical Chemistry at Unicamp and coordinates the Laboratory of Pharmaceutical Research and Chemometrics (LabFarQui). Her major interests are related to chemometrics applied to the development of analytical methods based on liquid chromatography and vibrational spectroscopy and development of pharmaceutical products and processes according to the strategy of Quality by Design (QbD).

\section{References}

1. Delhaye, M.; Migeon, M.; Compt. Rend. Acad. Sci. 1966, 262, 702.

2. Louden, J. D. In Practical Raman Spectroscopy; Gardiner, D. J.; Graves, P. R., eds.; Springer-Verlag: Berlin Heidelberg, 1989, p. $119-151$.

3. Turrell, G.; Dhamelincourt, P. In Modern Techniques in Raman Spectroscopy; Laserna, J. J., ed.; John Wiley \& Sons Ltd: Chichester, UK, 1996, p. 109-142.

4. Newbury, D. E.; Ritchie, N. W. M.; J. Anal. At. Spectrom. 2013, $28,973$.

5. Newbury, D. E.; Ritchie, N. W. M.; Scanning 2013, 35, 141.

6. Coling, D.; Kachar, B.; Curr. Protoc. Neurosci. 2001, doi.org/10.1002/0471142301.ns0201s00.

7. Smekal, A.; Naturwissenschaften 1928, 16, 612.

8. Raman, C. V.; Krishna, K. S.; Nature 1928, 121, 501.

9. Landsberg, G.; Mendelstam, L.; Naturwissenschaften 1928, 16, 557.

10. Sala, O.; Bassi, D.; Santos, P. S.; Hase, Y.; Forneris, R. I. M. G.; Temperini, M. L. A.; Kawano, Y.; Espectroscopia Raman: Princípios e Aplicações; Fundação Valeparaibana de Ensino: São José dos Campos, Brasil, 1984.

11. Porto, S. P. S.; Wood, D. L.; J. Opt. Soc. Am. 1962, 52, 251.

12. Dierker, S. B.; Murray, C. A.; Legrange, J. D.; Schlotter, N. E.; Chem. Phys. Lett. 1987, 137, 453.

13. Schmid, T.; Dariz, P.; J. Raman Spectrosc. 2013, 44, 882.

14. Staniszewska, E.; Malek, K.; Kaszowska, Z.; J. Raman Spectrosc. 2013, 44, 1144.

15. Zhu, X.; Xu, T.; Lin, Q.; Duan, Y.; Appl. Spectrosc. Rev. 2014, $49,64$. 
16. Strehle, K. R.; Rösch, P.; Berg, D.; Schulz, H.; Popp, J.; J. Agric. Food Chem. 2006, 54, 7020.

17. Vítek, P.; Novotná, K.; Hodaňová, P.; Rapantová, B.; Klem, K.; Spectrochim. Acta, Part A. 2017, 170, 234.

18. Stone, A.; Sakakura, M.; Shimotsuma, Y.; Stone, G.; Gupta, P.; Miura, K.; Hirao, K.; Dierolf, V.; Jain, H.; J. Non-Cryst. Solids 2010, 356, 3059.

19. Sharma, S. K.; Porter, J. N.; Misra, A. K.; Helsley, C. E.; Bates, D. E.; Eur. J. Mineral. 2013, 25, 715.

20. Doughty, D. C.; Hill, S. C.; J. Quant. Spectrosc. Radiat. Transfer 2017, 188, 103

21. Stewart, S.; Priore, R. J.; Nelson, M. P.; Treado, P. J.; Annu. Rev. Anal. Chem. 2012, 5, 337.

22. Sacré, P. Y.; Lebrun, P.; Chavez, P. F.; de Bleye, C.; Netchacovitch, L.; Rozet, E.; Klinkenberg, R.; Streel, B.; Hubert, P.; Ziemons, E.; Anal. Chim. Acta 2014, 818, 7.

23. Woo, D. J.; Sneed, B.; Peerally, F.; Heer, F. C.; Brewer, L. N.; Hooper, J. P.; Oswald, S.; Carbon 2013, 63, 404.

24. Guo, X.; Bi, H.; Zafar, A.; Liang, Z.; Shi, Z.; Sun, L.; Ni, Z.; Nanotechnology 2016, 27, 55702.

25. Chaturvedi, D.; Balaji, S.; Bn, V.; Ariese, F.; Umapathy, S.; Rangarajan, A.; Biosensors 2016, 6, 57.

26. Piqueras, S.; Duponchel, L.; Tauler, R.; de Juan, A.; Anal. Chim. Acta 2014, 819, 15.

27. Smith, G. P. S.; McGoverin, C. M.; Fraser, S. J.; Gordon, K. C.; Adv. Drug Delivery Rev. 2015, 89, 21.

28. Coltup, N. B.; Daly, L. H.; Wiberley, S. E.: Introduction to Infrared and Raman Spectroscopy; $3^{\text {rd }}$ ed.; Academic Press: San Diego, USA, 1990.

29. Käppler, A.; Fischer, D.; Oberbeckmann, S.; Schernewski, G.; Labrenz, M.; Eichhorn, K.-J.; Voit, B.; Anal. Bioanal. Chem. 2016, 408, 8377.

30. El-Diasty, F.; Vib. Spectrosc. 2011, 55, 1.

31. Smith, E.; Dent, G.; Modern Raman Spectroscopy - A Practical Approach; John Wiley \& Sons, Ltd: Chichester, UK, 2004

32. Pieczonka, N. P. W.; Aroca, R. F.; Chem. Soc. Rev. 2008, 37, 946.

33. Liu, R.; Liu, J.; Zhou, X.; Jiang, G.; Liu, R.; TrAC, Trends Anal. Chem. 2011, 30, 1462.

34. Zhang, Y.; Hong, H.; Myklejord, D. V.; Cai, W.; Small 2011, 7, 3261.

35. Qian, X.-M.; Nie, S. M.; Chem. Soc. Rev. 2008, 37, 912.

36. Guerrini, L.; Graham, D.; Chem. Soc. Rev. 2012, 41, 7085.

37. McNay, G.; Eustace, D.; Smith, W. E.; Faulds, K.; Graham, D.; Appl. Spectrosc. 2011, 65, 825.

38. Schlücker, S.; Angew. Chem., Int. Ed.2014, 53, 4756.

39. Wessel, J.; J. Opt. Soc. Am. B 1985, 2, 1538.

40. Pettinger, B.; Schambach, P.; Villagómez, C. J.; Scott, N.; Annu. Rev. Phys. Chem. 2012, 63, 379.

41. Sharma, G.; Deckert-Gaudig, T.; Deckert, V.; Adv. Drug Delivery Rev. 2015, 89, 42.
42. Kumar, N.; Mignuzzi, S.; Su, W.; Roy, D.; EPJ Tech. Instrum. 2015, 2, 9 .

43. Efremov, E. V.; Ariese, F.; Gooijer, C.; Anal. Chim. Acta 2008, 606, 119 .

44. Winterhalder, M. J.; Zumbusch, A.; Adv. Drug Delivery Rev. 2015, 89, 135.

45. Opilik, L.; Schmid, T.; Zenobi, R.; Annu. Rev. Anal. Chem. 2013, 6, 379.

46. Sacré, P. Y.; de Bleye, C.; Chavez, P. F.; Netchacovitch, L.; Hubert, P.; Ziemons, E.; J. Pharm. Biomed. Anal. 2014, 101, 123.

47. Zhang, L.; Henson, M. J.; Appl. Spectrosc. 2007, 61, 1015.

48. Eilers, P. H. C.; Boelens, H. F. M.; Leiden University Medical Centre Report 2005, https://zanran_storage.s3.amazonaws.com/ www.science.uva.nl/ContentPages/443199618.pdf, accessed in May 2019.

49. Vidal, M.; Amigo, J. M.; Chemom. Intell. Lab. Syst. 2012, 117, 138.

50. Childres, I.; Jauregui, L.; Park, W.; Cao, H.; Chen, Y. In New Developments in Photon and Materials Research; Jang, J, I., ed.; Nova Science Publishers: Hauppauge, USA, 2013, ch. 19.

51. Meier, R. J.; Vib. Spectrosc. 2005, 39, 266.

52. Miller, J. N.; Miller, J. C.; Statistics and Chemometrics for Analytical Chemistry, $6^{\text {th }}$ ed.; Pearson Publishing: Gosport, UK, 2010.

53. Bro, R.; Smilde, A. K.; Anal. Methods 2014, 6, 2812.

54. Wold, S.; Esbensen, K.; Geladi, P.; Chemom. Intell. Lab. Syst. 1987, 2, 37

55. Nascimento, J. M. P.; Dias, J. M. B.; IEEE Trans. Geosci. Remote Sens. 2005, 43, 898.

56. Chew, W.; Widjaja, E.; Garland, M.; Organometallics 2002, 21, 1982.

57. Gendrin, C.; Roggo, Y.; Collet, C.; J. Pharm. Biomed. Anal. 2008, 48, 533.

58. de Juan, A.; Jaumot, J.; Tauler, R.; Anal. Methods 2014, 6, 4964.

59. Geladi, P.; Kowalski, B. R.; Anal. Chim. Acta 1986, 185, 1.

60. Ballabio, D.; Consonni, V.; Anal. Methods 2013, 5, 3790.

61. Papageridis, K. N.; Siakavelas, G.; Charisiou, N. D.; Avraam, D. G.; Tzounis, L.; Kousi, K.; Goula, M. A.; Fuel Process. Technol. 2016, 152, 156

62. Liu, D.; Fu, C.; Zhang, N.; Zhou, H.; Kuang, Y.; Electrochim. Acta 2016, 213, 291.

63. Vogel, C.; Adam, C.; McNaughton, D.; Appl. Spectrosc. 2013, 67, 1101.

64. Qin, J.; Chao, K.; Kim, M. S.; Postharvest Biol. Technol. 2012, $71,21$.

65. Matousek, P.; Clark, I. P.; Draper, E. R. C.; Morris, M. D.; Goodship, A. E.; Everall, N.; Towrie, M.; Finney, W. F.; Parker, A. W.; Appl. Spectrosc. 2005, 59, 393. 
66. Conti, C.; Realini, M.; Colombo, C.; Sowoidnich, K.; Afseth, N. K.; Bertasa, M.; Botteon, A.; Matousek, P.; Anal. Chem. 2015, 87, 5810.

67. Wang, P.; Pang, S.; Pearson, B.; Chujo, Y.; McLandsborough, L.; Fan, M.; He, L.; Anal. Bioanal. Chem. 2017, 409, 2229.

68. Lamas, A.; Miranda, J. M.; Regal, P.; Vázquez, B.; Franco, C. M.; Cepeda, A.; Microbiol. Res. 2018, 206, 60.

69. Zhang, X.; de Juan, A.; Tauler, R.; Appl. Spectrosc. 2015, 69, 993.

70. Yaseen, T.; Sun, D.-W.; Cheng, J.-H.; Trends Food Sci. Technol. 2017, 62, 177.

71. Batonneau, Y.; Sobanska, S.; Laureyns, J.; Bremard, C.; Environ. Sci. Technol. 2006, 40, 1300.

72. Konorov, S. O.; Schulze, H. G.; Piret, J. M.; Aparicio, S. A.; Turner, R. F. B.; Blades, M. W.; Appl. Spectrosc. 2011, 65, 1009.

73. Han, G.; Liu, R.; Han, M.-Y.; Jiang, C.; Wang, J.; Du, S.; Liu, B.; Zhang, Z.; Anal. Chem. 2014, 86, 11503.

74. Marini, M.; Das, G.; La Rocca, R.; Gentile, F.; Limongi, T.; Santoriello, S.; Scarpellini, A.; di Fabrizio, E.; Microelectron. Eng. 2014, 119, 151.

75. Saar, B. G.; Freudiger, C. W.; Reichman, J.; Stanley, C. M.; Holtom, G. R.; Xie, X. S.; Science 2010, 330, 1368.

76. Masia, F.; Karuna, A.; Borri, P.; Langbein, W.; J. Raman Spectrosc. 2015, 46, 727.

77. Krafft, C.; Schmitt, M.; Schie, I. W.; Cialla-May, D.; Matthäus, C.; Bocklitz, T.; Popp, J.; Angew. Chem., Int. Ed. 2017, 56, 4392.

78. Jermyn, M.; Desroches, J.; Aubertin, K.; St-Arnaud, K.; Madore, W.-J.; De Montigny, E.; Guiot, M.-C.; Trudel, D.; Wilson, B. C.; Petrecca, K.; Leblond, F.; Phys. Med. Biol. 2016, 61, R370.

79. Duarte, A. S.; Schnedermann, C.; Kukura, P.; Sci. Rep. 2016, 6, 37516 .

80. Snitka, V.; Rodrigues, R. D.; Lendraitis, V.; Microelectron. Eng. 2011, 88, 2759.

81. Deckert-Gaudig, T.; Richter, M.; Knebel, D.; Jähnke, T.; Jankowski, T.; Stock, E.; Deckert, V.; Appl. Spectrosc. 2014, $68,916$.
82. Schulze, H. G.; Turner, R. F. B.; Appl. Spectrosc. 2014, 68, 185.

83. Wang, Y.-P.; Wang, Y.; Spencer, P.; Appl. Spectrosc. 2006, 60, 826.

84. Qin, J.; Kim, M. S.; Schmidt, W. F.; Cho, B.-K.; Peng, Y.; Chao, K.; J. Raman Spectrosc. 2016, 47, 437.

85. Chou, I.-M.; Wang, A.; J. Asian Earth Sci. 2017, 145, 309.

86. Wang, A.; Korotev, R. L.; Jolliff, B. L.; Ling, Z.; Planet. Space Sci. 2015, 112, 23.

87. Frosch, T.; Tarcea, N.; Schmitt, M.; Thiele, H.; Langenhorst, F.; Popp, J.; Anal. Chem. 2007, 79, 1101.

88. García-Florentino, C.; Maguregui, M.; Morillas, H.; Balziskueta, U.; Azcarate, A.; Arana, G.; Madariaga, J. M.; J. Raman Spectrosc. 2016, 47, 1458.

89. Botteon, A.; Conti, C.; Realini, M.; Colombo, C.; Matousek, P.; Anal. Chem. 2017, 89, 792.

90. Eder, G. C.; Spoljaric-Lukacic, L.; Chernev, B. S.; Anal. Bioanal. Chem. 2012, 403, 683.

91. Kazemi-Zanjani, N.; Kergrene, E.; Liu, L.; Sham, T.-K.; Lagugné-Labarthet, F.; Sensors 2013, 13, 12744.

92. de Farias, R. F.; Introdução à Química Forense, $2^{\mathrm{a}}$ ed.; Editora Átomo: Campinas, Brasil, 2008.

93. Deng, S.; Liu, L.; Liu, Z.; Shen, Z.; Li, G.; He, Y.; Appl. Opt. 2012, 51, 3701.

94. Borba, F. D. S. L.; Jawhari, T.; Saldanha Honorato, R.; de Juan, A.; Analyst 2017, 142, 1106.

95. Hufziger, K. T.; Bykov, S. V.; Asher, S. A.; Appl. Spectrosc. 2017, 71, 173.

96. Scoutaris, N.; Vithani, K.; Slipper, I.; Chowdhry, B.; Douroumis, D.; Int. J. Pharm. 2014, 470, 88.

97. Tolbert, D.; Cloyd, J.; Biton, V.; Bekersky, I.; Walzer, M.; Wesche, D.; Drummond, R.; Lee, D.; Epilepsia 2015, 56, 915.

98. Mitsutake, H.; Castro, S. R.; de Paula, E.; Poppi, R. J.; Rutledge, D. N.; Breitkreitz, M. C.; Int. J. Pharm. 2018, 552, 119.

Submitted: February 28, 2019

Published online: May 24, 2019 